\title{
Quantitative analysis of patellar tendon size and structure in asymptomatic professional players: sonographic study
}

\author{
Maurizio Giacchino ${ }^{1}$ \\ Cristina Caresio ${ }^{2}$ \\ Negar E. Gorji ${ }^{3}$ \\ Filippo Molinari² \\ Giuseppe Massazza ${ }^{4}$ \\ Marco Alessandro Minetto 4
}

1 Medical Lab, Asti, Italy

2 Biolab, Department of Electronics and Telecommunications, Politecnico di Torino, Turin, Italy

3 Division of Endocrinology, Diabetology and Metabolism, Department of Medical Sciences, University of Turin, Turin, Italy

4 Division of Physical Medicine and Rehabilitation, Department of Surgical Sciences, University of Turin, Turin, Italy

Corresponding Author:

Marco Alessandro Minetto

Department of Surgical Sciences,

University of Turin

Corso Dogliotti 14

10126 Turin, Italy

E-mail: marco.minetto@unito.it

\section{Summary}

Background: Ultrasonographic abnormalities of the patellar tendon frequently occur in asymptomatic athletes and it is not always clear whether they precede (and may predict) the development of tendinopathy.

Objective: This study aimed to investigate by ultrasonography the prevalence of patellar tendon abnormalities in players of "pallapugno" and to establish whether structural tendon abnormalities predict tendinopathy development.

Methods: Ultrasound B-mode images of the patellar tendon of both sides were acquired in fourteen throwers. Qualitative assessments of tendon structure and neovascularization and quantitative assessments of tendon thickness, cross sectional area (CSA), and echo-intensity were performed.

Results: Qualitative assessments showed a subclinical tendinopathy of the non-dominant tendon in 5 out of 14 throwers (35\% of cases), while quantitative assessments showed abnormalities of the nondominant tendon in 8 out of 14 players $(57 \%$ of cases). Echo-intensity and CSA were the quantitative variables most discriminant between asymptomatic players without structural tendon abnormalities and those with tendon abnormalities. Two players ( 2 out of 8 cases: $25 \%$ ) developed a clinical tendinopathy after a follow-up of six months.

Conclusion: The prevalence of subclinical tendinopathy in the non-dominant patellar tendon of throwers was high. Patellar tendon abnormalities at baseline seem to increase the risk of development of subsequent patellar tendinopathy.

Level of evidence: II b (individual cohort study).

KEY WORDS: patellar tendon, tendinopathy, thrower players, quantitative ultrasonography.

\section{Introduction}

Musculoskeletal ultrasonography is an effective technique to visualize normal and pathological tendons and to non-invasively evaluate (qualitative assessment) ${ }^{1,2}$ or measure (quantitative analysis) parameters reflecting tendon size (length, thickness, and cross-sectional area) and structure (echo-intensity) ${ }^{3-6}$. Patellar tendon ultrasonography is commonly performed in the routine management of athletes of different disciplines to investigate tendon abnormalities that may occur due to repetitive tendon overload ${ }^{7-10}$. In fact, patellar tendinopathy is a common overuse disorder typically occurring in athletes who participate in sports that require jumping, including volleyball and basketball, hence the label "jumper's knee"11,12. However, ultrasonographic tendon abnormalities (such as hypoechoic areas, increased tendon thickness, neovascularization) have been identified in large percentages of asymptomatic athletes $8,10,13$, therefore it is not always clear whether structural tendon abnormalities precede and predict the development of functional tendon abnormalities and tendon pain.

The aims of the present study were to: I) investigate the prevalence of patellar tendon abnormalities in elite players of "pallapugno", a game practiced in the north-west of Italy (similar to frisian handball, Basque pelota, and French game called "ballon au poing") and that implies in throwers a repetitive overload of the non-dominant lower limb; II) compare the diagnostic accuracy of qualitative to the accuracy of quantitative assessments of tendon size and structure in detecting structural tendon abnormalities; III) establish whether structural patellar tendon abnormalities predict tendinopathy development. 


\section{Materials and methods}

\section{Subjects}

Fourteen male throwers (age, mean \pm SD: $21.8 \pm 4.2$ years) volunteered to participate in the study. They were free from neuromuscular or skeletal impairments and were asked to refrain from performing strenuous physical activity during $24 \mathrm{~h}$ before the experimental session. Side dominance was assessed with the "Waterloo Handedness and Footedness Questionnaires - Revised"14. All subjects were right side dominant. Before participating to the study, the subjects received a detailed explanation of the proto$\mathrm{col}$ and gave written informed consent. The study conformed to the guidelines of the Declaration of Helsinki, met the ethical standards of the journal ${ }^{15}$ and was approved by the local ethics committee.

\section{Procedures}

Ultrasound B-mode images of the patellar tendon were acquired in each subject during a single experimental session. Both sides were investigated.

The same experienced sonographer (MG) performed all assessments. Twenty scans were acquired in total for each subject while he was lying in a supine position and with the quadriceps muscle relaxed: 12 scans were acquired with a knee angle of $0^{\circ}$ (fully extended knee), while 8 scans were acquired with a knee angle of $30^{\circ}$ (Tab. I).

In addition, ultrasound analysis and qualitative assessments of tendon structure and neovascularization (see below) were repeated 6 months after the first investigation in players who became symptomatic for patellar tendinopathy.

\section{Ultrasound equipment}

All measurements were performed using a ClearVue 550 ultrasound device (Philips Medical Systems, MiIan, Italy) equipped by a linear-array transducer with variable frequency band (4-12 MHz). Gain was set at $50 \%$ of the range, dynamic image compression was turned off, and time gain compensation was maintained in the same (neutral) position for all scans. Power Doppler imaging was performed with slow perfusion settings. All system-setting parameters were kept constant throughout the study and for each subject. Pictures were stored as DICOM files and transferred to a computer for processing.

Table I. Acquisitions and analyses performed in each subject for the qualltative assessments of patellar tendon structure and neovascularization and for the quantitative assessments of tendon thickness, cross sectional area (CSA), and echo-intensity. PD: Power Doppler.

\begin{tabular}{|c|c|c|}
\hline Images & Acquisition & Analyses \\
\hline \multicolumn{3}{|c|}{ Knee angle: $0^{\circ}$} \\
\hline $\begin{array}{l}\text { Image I } \\
\text { (Image 7) }\end{array}$ & $\begin{array}{c}\text { Transversal plane, proximal probe position, } \\
\text { non-dominant (dominant) side }\end{array}$ & $\begin{array}{c}\text { CSA and echo-intensity quantitative analyses } \\
\text { Structure qualitative analysis }\end{array}$ \\
\hline $\begin{array}{l}\text { Image } 2 \\
\text { (Image 8) }\end{array}$ & $\begin{array}{c}\text { Transversal plane, proximal probe position, } \\
\text { non-dominant (dominant) side (including PD } \\
\text { registration) }\end{array}$ & Neovascularization qualitative analysis \\
\hline $\begin{array}{l}\text { Image } 3 \\
\text { (Image 9) }\end{array}$ & $\begin{array}{l}\text { Longitudinal plane, proximal probe position, } \\
\text { non-dominant (dominant) side }\end{array}$ & $\begin{array}{l}\text { Thickness and echo-intensity quantitative analyses } \\
\text { Structure qualitative analysis }\end{array}$ \\
\hline $\begin{array}{l}\text { Image } 4 \\
\text { (Image 10) }\end{array}$ & $\begin{array}{l}\text { Longitudinal plane, proximal probe position, } \\
\text { non-dominant (dominant) side (including PD } \\
\text { registration) }\end{array}$ & Neovascularization qualitative analysis \\
\hline $\begin{array}{l}\text { Image } 5 \\
\text { (Image 11) }\end{array}$ & $\begin{array}{l}\text { Transversal plane, central probe position, } \\
\text { non-dominant (dominant) side }\end{array}$ & CSA and echo-intensity quantitative analyses \\
\hline $\begin{array}{l}\text { Image } 6 \\
\text { (Image 12) }\end{array}$ & $\begin{array}{l}\text { Longitudinal plane, central probe position, } \\
\text { non-dominant (dominant) side }\end{array}$ & Thickness and echo-intensity quantitative analyses \\
\hline \multicolumn{3}{|c|}{ Knee angle: $30^{\circ}$} \\
\hline $\begin{array}{l}\text { Image 13 } \\
\text { (Image 17) }\end{array}$ & $\begin{array}{l}\text { Transversal plane, proximal probe position, } \\
\text { non-dominant (dominant) side }\end{array}$ & Structure qualitative analysis \\
\hline $\begin{array}{l}\text { Image } 14 \\
\text { (Image 18) }\end{array}$ & $\begin{array}{l}\text { Longitudinal plane, proximal probe position, } \\
\text { non-dominant (dominant) side }\end{array}$ & Structure qualitative analysis \\
\hline $\begin{array}{l}\text { Image } 15 \\
\text { (Image 19) }\end{array}$ & $\begin{array}{l}\text { Transversal plane, central probe position, } \\
\text { non-dominant (dominant) side }\end{array}$ & Structure qualitative analysis \\
\hline $\begin{array}{l}\text { Image } 16 \\
\text { (Image 20) }\end{array}$ & $\begin{array}{l}\text { Longitudinal plane, central probe position, } \\
\text { non-dominant (dominant) side }\end{array}$ & Structure qualitative analysis \\
\hline
\end{tabular}


Qualitative assessments of tendon structure and neovascularization

The same experienced sonographer (MG) who performed the image acquisitions performed also the qualitative assessments.

Tendon structure was evaluated both in the longitudinal and in the transversal scans by using the four-

\section{Longitudinal scan: proximal thickness}

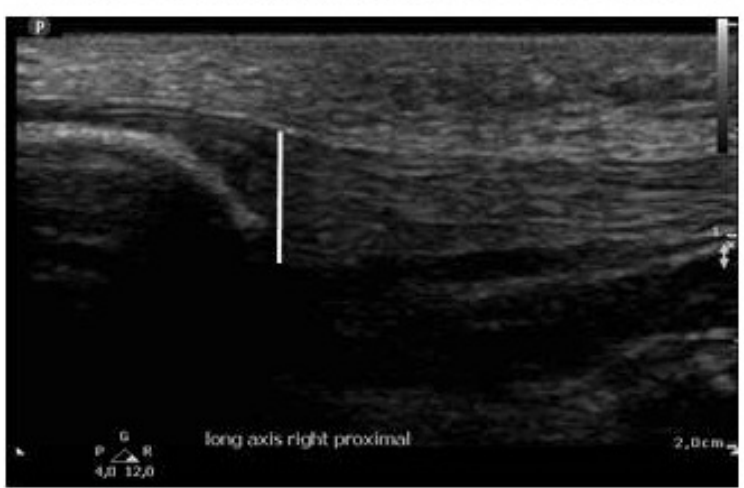

\section{Longitudinal scan: proximal ROI}

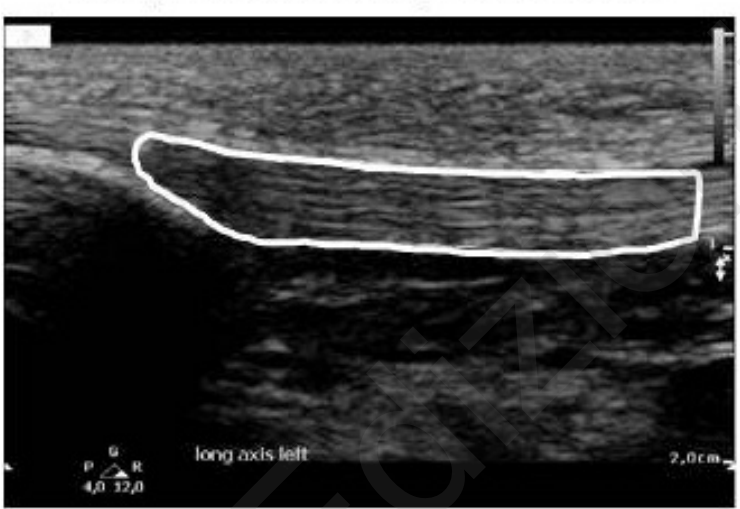

Transversal scan: proximal ROI

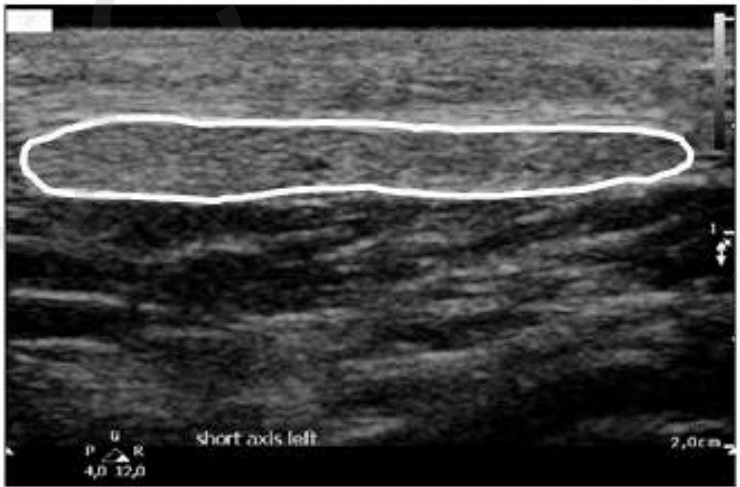

grade scale proposed by Sunding et al. ${ }^{16}: 0$ : normal structure (homogeneous echogenicity); 1: light structural changes (discrete hypo-echogenic areas); 2: moderate structural changes (some well-defined hypo-echogenic areas); 3: severe structural changes (extended hypo-echogenic areas).

Neovascularization was evaluated both in the longitu-

\section{Longitudinal scan: central thickness}

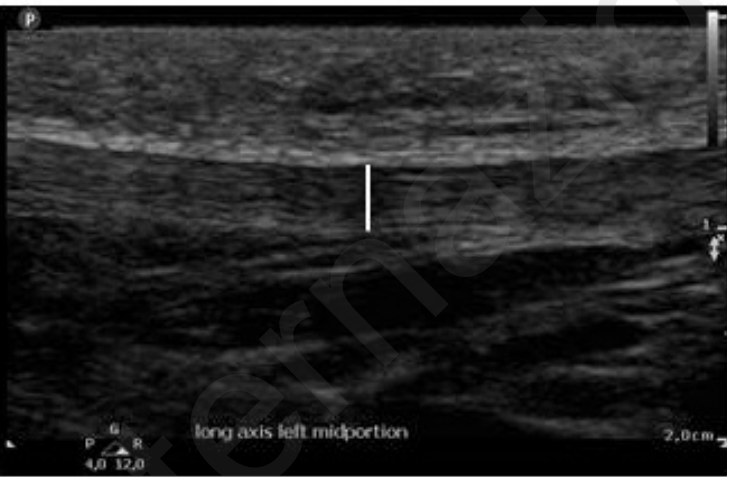

\section{Longitudinal scan: central ROI}

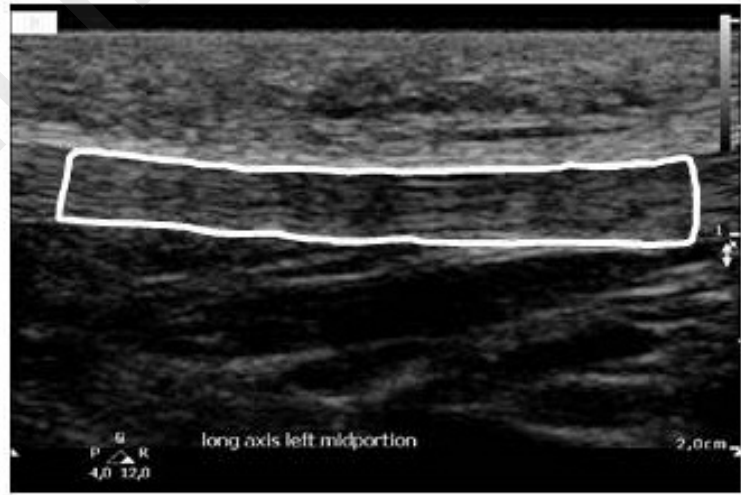

\section{Transversal scan: central ROI}

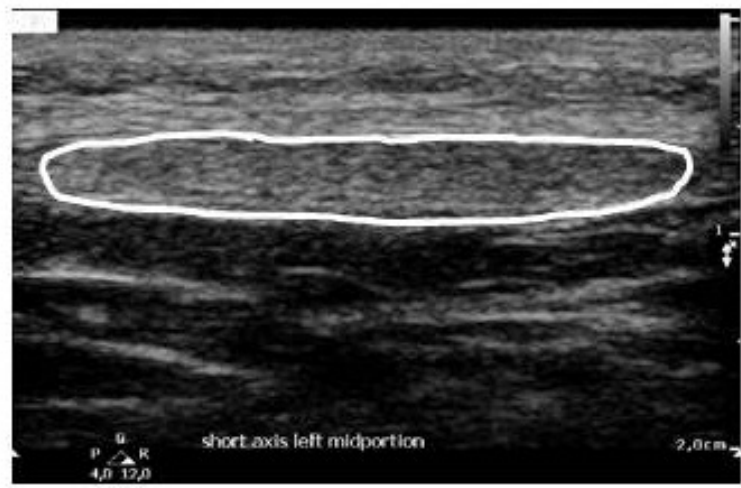

Figure 1. Representative patellar tendon scans showing the targets for the thickness measurement (upper panels), longitudinal regions of interest (middle panels), and transversal regions of interest (lower panels) for the echo-intensity measurements: ROI: region of interest. 
dinal and in the transversal scans by using the fourgrade scale proposed by Sunding et al. ${ }^{16}$ : 0 : no neovascularization; 1 : mild neovascularization (a few solitary blood vessels); 2: moderate neovascularization (moderate quantity, mostly transversal blood vessels); 3: severe neovascularization (several, mostly horizontal blood vessels spread in the whole depth of the tendon).

We arbitrarily defined "subclinical tendinopathy" as the presence of either light structural changes in association with at least mild neovascularization or moderate/severe structural changes with/without neovascularization.

\section{Measurement of tendon thickness}

Tendon thickness was measured in the longitudinal scans acquired with 0-degree knee angle: as shown in the representative images in Figure 1 (upper panels), the target for the thickness measurement was the thickest part of the tendon both for the proximal probe position (i.e., just below the apex of the patella: left panel) and for the central probe position (i.e., midportion of the tendon: right panel).

\section{Measurement of tendon cross sectional area}

Tendon CSA was measured in the transversal scans acquired with 0-degree knee angle: as shown in the representative images in Figure 1 (lower panels), a region of interest was chosen in each scan to include as much of the tendon as possible. The area was determined for the selected region of interest by a custom software developed in MATLAB (The MathWorks, Inc., Natick, MA, USA).

\section{Measurement of tendon echo-intensity}

Tendon echo-intensity (for images acquired with 0degree knee angle) was measured both in the longitudinal (Fig. 1: middle panels) and in the transversal scan (Fig. 1: lower panels) regions of interest: the mean echo intensity (8-bit resolution, resulting in a number between 0 and 255, where black $=0$, white $=255$ ) was determined by a custom software developed in MATLAB (The MathWorks, Inc., Natick, MA, USA).

\section{Statistical analysis}

Since the Shapiro-Wilk test for the normal distribution of the data failed, non-parametric tests were used. The Friedman's ANOVA followed by Dunn's post-hoc test and the Wilcoxon test were adopted for comparisons of size parameters (i.e., thickness and cross sectional area) and echo-intensity both between the two tendon portions investigated (proximal vs central) and between the two sides (left $v s$ right).

$\mathrm{K}$-means cluster analysis followed by F-ratio calculation were applied to the side-to-side differences in size and echo-intensity of the tendon proximal portion in order to discern between different groups of players.
Data are expressed as median (and range) and are represented as box-and-whisker plots. Threshold for statistical significance was set at $P<0.05$. Statistical tests were performed with the IBM SPSS Statistics (version 20 - IBM Corporation, Armonk, NY, USA) software package.

\section{Results}

Qualitative assessments of tendon structure and neovascularization

Figure 2 reports a representative example of one thrower player showing in both sides a normal structure of the patellar tendon without neovascularization, while Figure 3 shows for another representative thrower player moderate structural changes and mild neovascularization in the proximal portion of the left patellar tendon and normal structure in the homologous portion of the contralateral tendon.

Similar to these representative examples, the qualitative assessment of the longitudinal and transversal scans of all players showed normal structure without neovascularization in 7 out of 14 throwers and structural changes with or without neovasculation in the proximal portion of the non-dominant tendon of the other 7 throwers. The structure and neovascularization scores of the non-dominant tendon of all players are listed in Table II: in total, 5 out of 14 throwers (35\% of cases: \# 4-8-9-11-13 in Tab. II) were considered affected by "subclinical tendinopathy" of the non-dominant tendon because of the presence of light structural changes in association with at least mild neovascularization (two out of five players) or moderate/severe structural changes with or without neovascularization (three out of five players).

\section{Quantitative assessments of tendon size and echo-intensity}

Figure 4 reports the box-and-whisker plots of the tendon size parameters (thickness and CSA) and of the eco-intensity for both sides (left and right) and for both portions (proximal and central) investigated.

As expected, the thickness of the proximal portion of both sides of the tendon was higher compared to that of the central portion (left side: $\mathrm{P}<0.001$; right side: $P<0.01$ ), while no differences were observed in tendon CSA and echo-intensity between the proximal and central portion of both sides ( $P$ values $>0.05$ ). Further, no differences in thickness, CSA, and echointensity of the tendon proximal portion were observed between the two sides ( $P$ values $>0.05)$.

Analysis of individual data and K-means cluster analysis unraveled a remarkable interindividual variability in the side-to-side differences in size and echointensity of the tendon proximal portion. In fact, 6 out of 14 players ( $43 \%$ of cases) presenting comparable values of thickness, CSA, and echo-intensity between the proximal portion of the two sides were classified in cluster 1 (Tab. III: $P \geq 0.05$ for all side-to-side comparisons), while 8 out of 14 players (57\% of cases) 


\section{LEFT PATELLAR TENDON}

\section{RIGHT PATELLAR TENDON}

\section{Longitudinal scan, 0-degree knee angle}
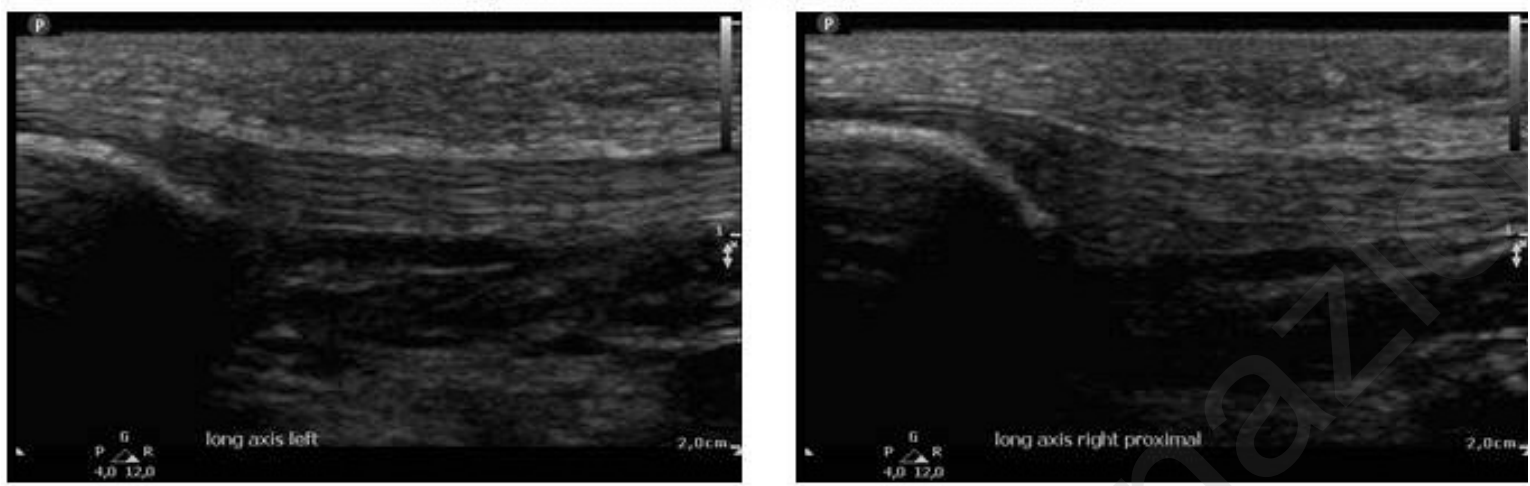

\section{Longitudinal scan, 0-degree knee angle, Power Doppler analysis}
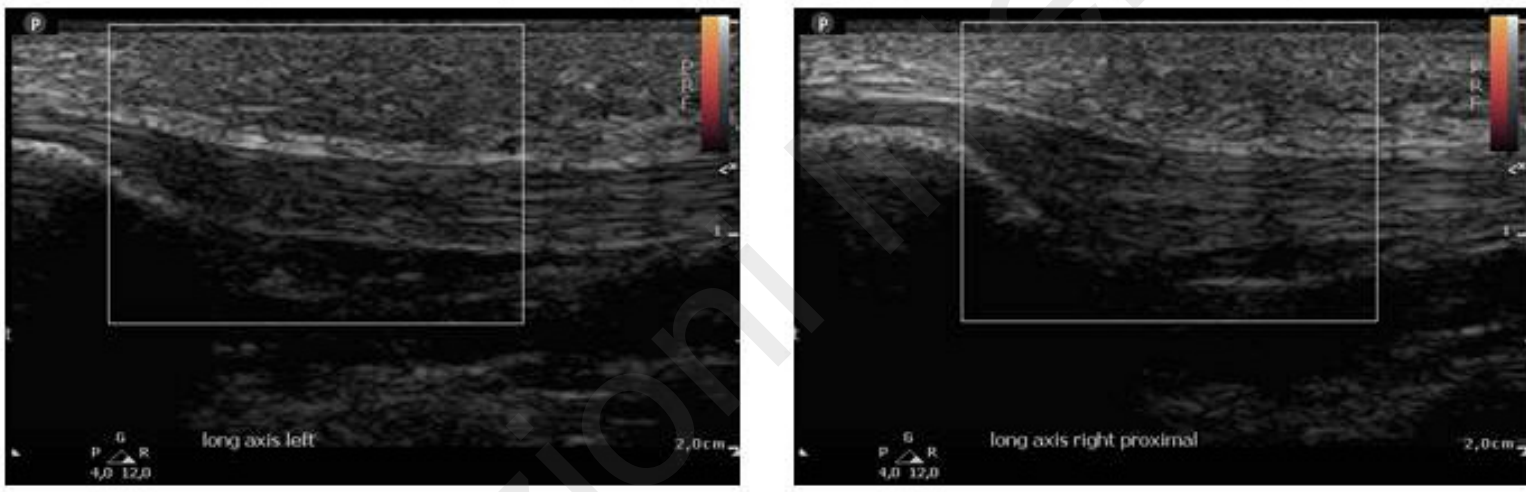

\section{Transversal scan, 0 -degree knee angle}
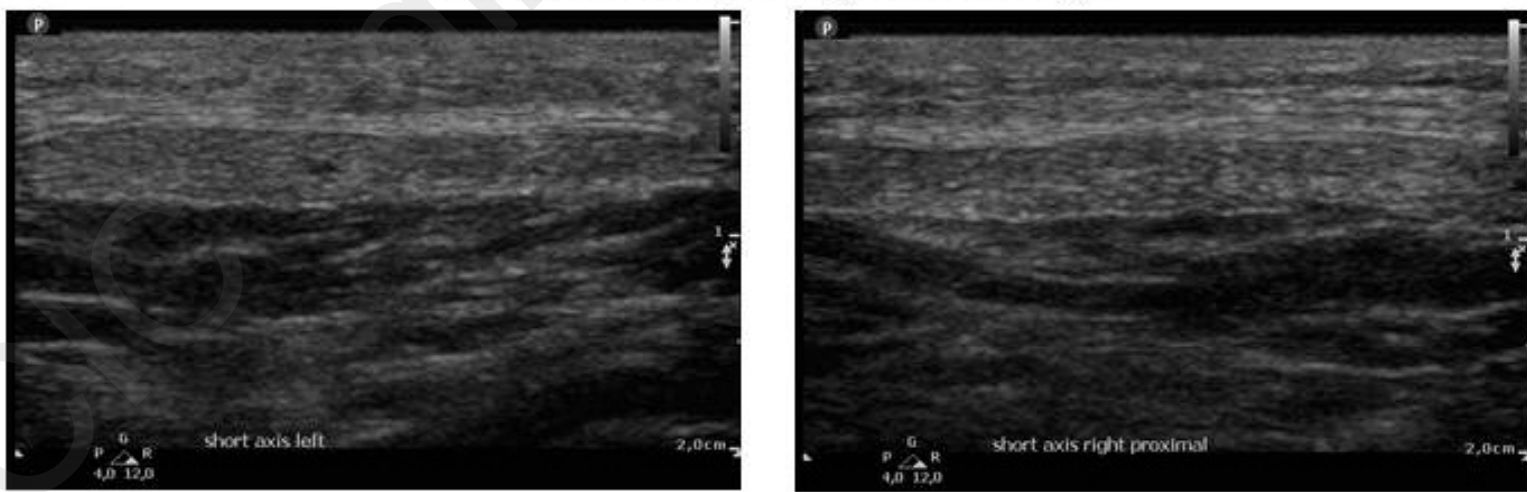

Figure 2. Representative patellar tendon scans of one thrower player showing in both sides a normal structure of the tendon without neovascularization.

presenting side-to-side differences in CSA $(P=0.01)$ and echo-intensity $(\mathrm{P}=0.01)$ were included in cluster 2 (Tab. III: $\mathrm{P}<0.05$ for side-to-side comparisons in CSA and echo-intensity). In this subgroup of 8 players, CSA was significantly higher and echo-intensity was significantly lower in the non-dominant tendon compared to the dominant one. The following significances (relative to the F-ratio for each variable) were obtained for the differences between the two clusters: thickness: $P=0.165$; CSA: $P=0.005$; longitudinal echo- 


\section{LEFT PATELLAR TENDON}

\section{RIGHT PATELLAR TENDON}

\section{Longitudinal scan, 0-degree knee angle}
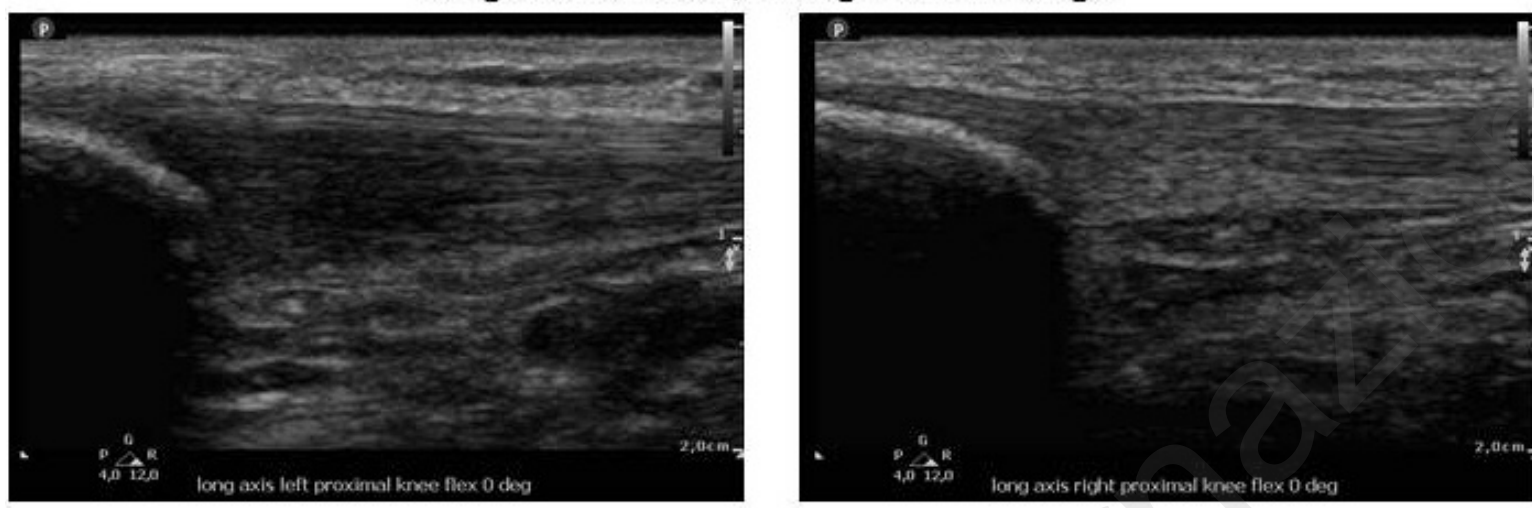

\section{Longitudinal scan, 0-degree knee angle, Power Doppler analysis}
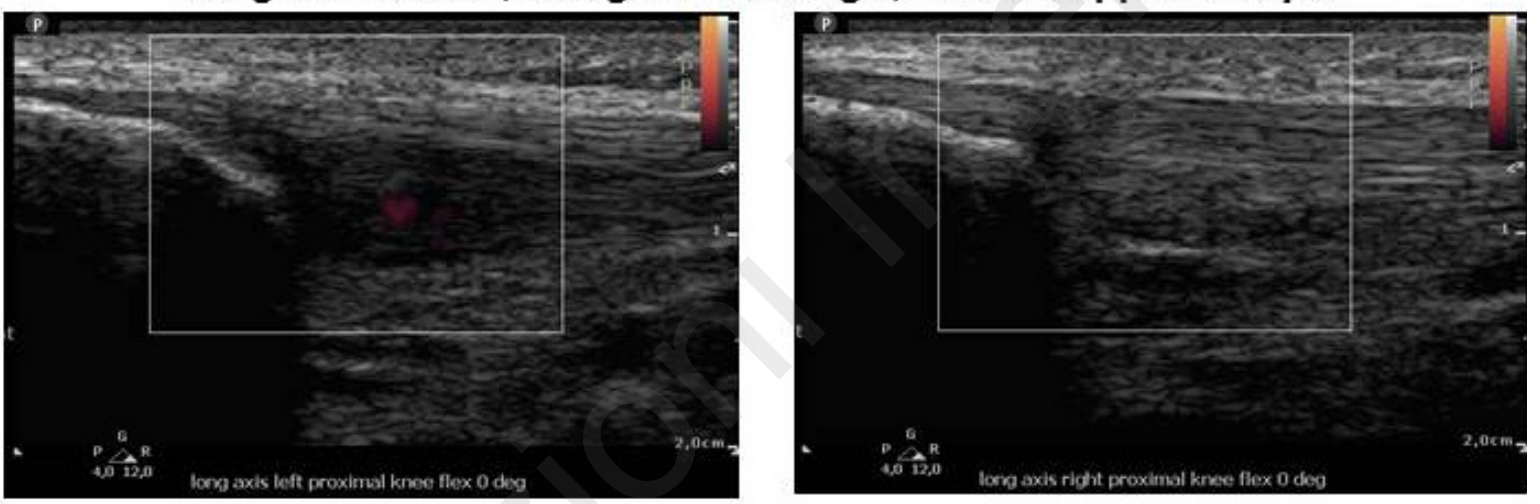

\section{Transversal scan, 0 -degree knee angle}
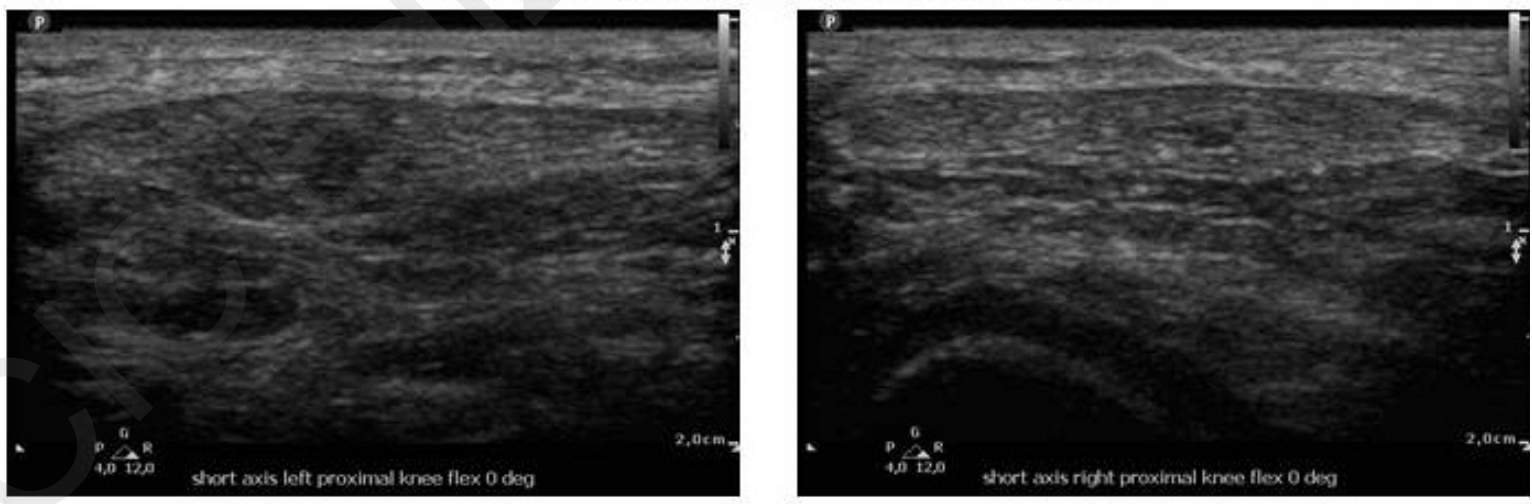

Figure 3. Representative patellar tendon scans of one thrower player showing moderate structural changes and mild neovascularization in the proximal portion of the left patellar tendon and normal structure in the homologous portion of the right tendon.

intensity: $P=0.001$; transversal echo-intensity: $\mathrm{P}=0.003$.

Briefly, CSA and echo-intensity were the quantitative variables most discriminant between the two clusters and resulted significantly different between the dominant and the non-dominant tendon in a large subgroup ( $57 \%$ of cases) of asymptomatic thrower players. 
Table II. Results of the qualitative assessments of tendon structure and neovascularization (second and third column: 5 players affected by "subclinical tendinopathy" of the non-dominant patellar tendon are highlighted in bold) and of the cluster analysis applied to quantitative data of cross-sectional area and echointensity (fourth column). The last column reports that a clinical tendinopathy of the non-dominant patellar tendon was found in two players (\# 4 and 9) after a follow-up of six months.

\begin{tabular}{lllll}
\hline Player & $\begin{array}{l}\text { Structure } \\
\text { score }\end{array}$ & $\begin{array}{l}\text { Neovascu- } \\
\text { larisation } \\
\text { score }\end{array}$ & Cluster & Follow-up \\
\hline 1 & 0 & 0 & 1 & - \\
2 & 1 & 0 & 2 & - \\
3 & 0 & 0 & 2 & - \\
4 & 1 & 1 & 2 & + \\
5 & 0 & 0 & 1 & - \\
6 & 0 & 0 & 1 & - \\
7 & 0 & 0 & 1 & - \\
8 & 2 & 1 & 2 & - \\
9 & 2 & 1 & 2 & + \\
10 & 0 & 0 & 2 & - \\
11 & 1 & 1 & 2 & - \\
12 & 1 & 0 & 1 & - \\
13 & 2 & 0 & 2 & - \\
14 & 0 & 0 & 1 & - \\
\hline
\end{tabular}

Correspondence between qualitative and quantitative assessments

The 5 players identified by the qualitative analysis as affected by subclinical tendinopathy (\# 4-8-9-11-13 in Tab. II) were a subgroup of the 8 players classified in cluster 2.

Briefly, the qualitative assessments of tendon structure and neovascularization were in good agreement with the results of the cluster analysis applied to quantitative data of CSA and echo-intensity.

\section{Do patellar tendon abnormalities predict tendi-} nopathy?

Two players (\# 4 and 9 in Tab. II) identified by qualitative analysis as affected by subclinical tendinopathy and classified in cluster 2 ( 2 out of 8 cases: $25 \%$ ) developed a clinical tendinopathy (that was confirmed by ultrasonography through qualitative assessments of tendon structure and neovascularization) after a follow-up of six months, while no players classified in cluster 1 (0 out of 6 cases: 0\%) developed a tendinopathy (Tab. II, last column). Therefore, patellar tendon abnormalities at baseline seems to increase (although not significantly) the risk of development of subsequent patellar tendinopathy (relative risk $=3.89,95 \% \mathrm{Cl} 0.22-68.67, \mathrm{P}=0.35)$.

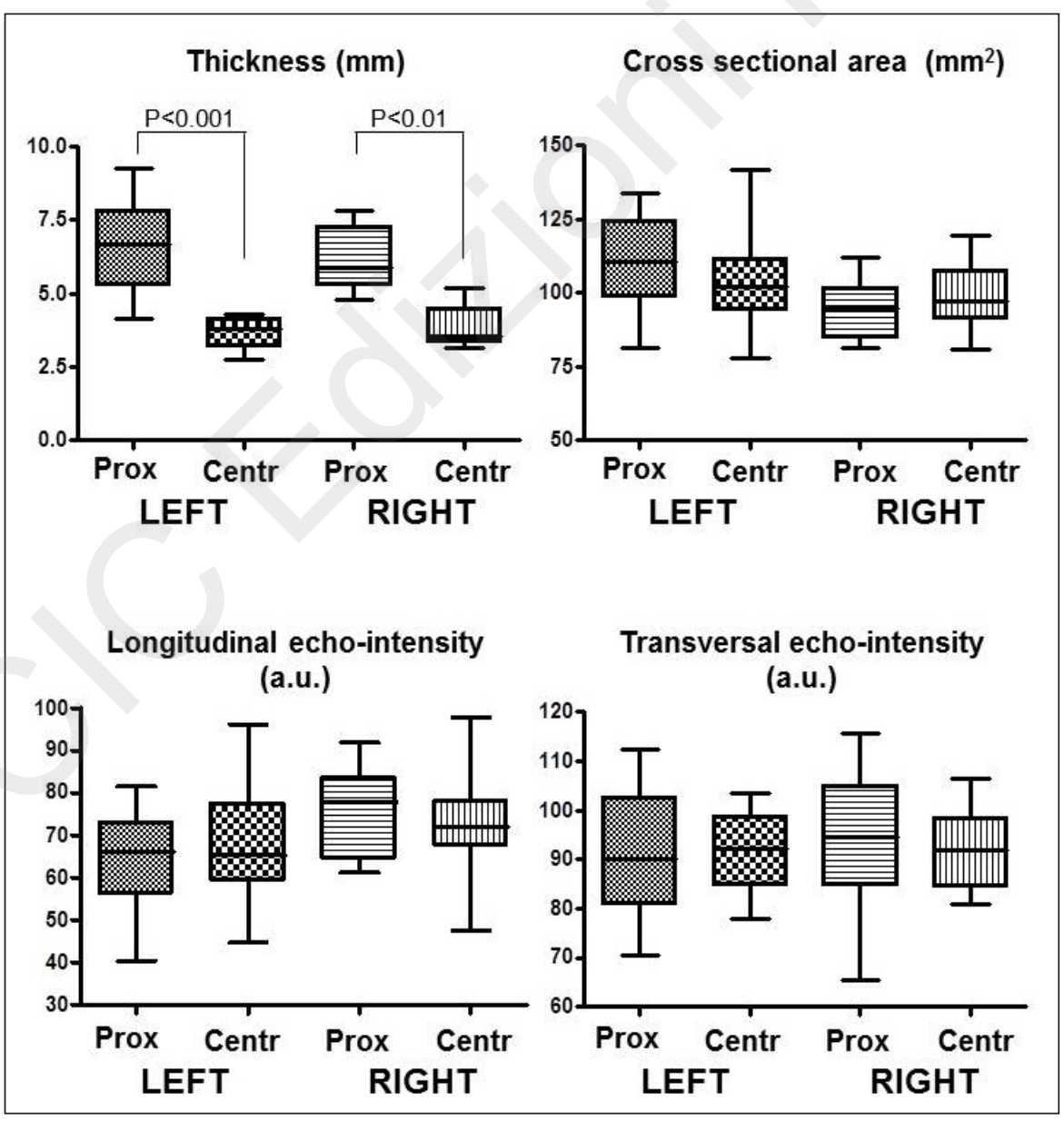

Figure 4. Patellar tendon thickness, cross sectional area, longitudinal echo-intensity, and transversal echo-intensity for both sides (left and right) and for both portions (proximal and central) investigated in the whole group of 14 thrower players. 
Table III. Median (min-max values) of the size parameters and echo-intensity for the tendon proximal portion of the right and left side. Statistically significant differences are highlighted in bold. CSA: cross sectional area, a.u.: arbitrary units.

\begin{tabular}{|c|c|c|c|}
\hline Variable & Left side & Right side & $P$ value \\
\hline \multicolumn{4}{|c|}{ Cluster 1 ( $n=6$ players) } \\
\hline Thickness (mm) & $\begin{array}{c}5.3 \\
(4.2-6.4)\end{array}$ & $\begin{array}{c}5.4 \\
(4.8-6.3)\end{array}$ & 0.75 \\
\hline $\operatorname{CSA}\left(\mathrm{mm}^{2}\right)$ & $\begin{array}{c}98.8 \\
(81.3-121.7)\end{array}$ & $\begin{array}{c}95.9 \\
(83.0-112.1)\end{array}$ & 0.92 \\
\hline Longitudinal echo-intensity (a.u.) & $\begin{array}{c}96.4) \\
(70.6-109.2\end{array}$ & $\begin{array}{c}91.4 \\
(65.6-107.6)\end{array}$ & 0.05 \\
\hline Transversal echo-intensity (a.u.) & $\begin{array}{c}73.2 \\
(55.3-81.4)\end{array}$ & $\begin{array}{c}64.3 \\
(61.2-68.1)\end{array}$ & 0.46 \\
\hline \multicolumn{4}{|c|}{ Cluster 2 ( $n=8$ players) } \\
\hline Thickness (mm) & $\begin{array}{c}7.7 \\
(5.6-9.3)\end{array}$ & $\begin{array}{c}6.8 \\
(5.3-7.8)\end{array}$ & 0.12 \\
\hline $\mathrm{CSA}\left(\mathrm{mm}^{2}\right)$ & $\begin{array}{c}120.3 \\
(107.2-133.8)\end{array}$ & $\begin{array}{c}93.4 \\
(81.2-109.1)\end{array}$ & 0.01 \\
\hline Longitudinal echo-intensity (a.u.) & $\begin{array}{c}84.0 \\
(74.9-112.5)\end{array}$ & $\begin{array}{c}94.6 \\
(85.1-115.5)\end{array}$ & 0.01 \\
\hline Transversal echo-intensity (a.u.) & $\begin{array}{c}63.1 \\
(40.3-70.6)\end{array}$ & $\begin{array}{c}80.1 \\
(77.0-91.9)\end{array}$ & 0.01 \\
\hline
\end{tabular}

\section{Discussion}

In the present study, patellar tendon ultrasound images were acquired from both sides of 14 asymptomatic thrower players and qualitative assessments (of tendon structure and neovascularization) and quantitative measurements (of tendon thickness, CSA, and echo-intensity) were performed.

Referring to the aims listed in the Introduction, the main results can be summarized as follows: I) qualitative assessments showed a subclinical tendinopathy of the non-dominant tendon in 5 out of 14 players (35\% of cases); II) quantitative assessments showed abnormalities of the non-dominant tendon in 8 out of 14 players (57\% of cases); III) qualitative assessments of tendon structure and neovascularization were in good agreement with the results of the cluster analysis applied to quantitative data of CSA and echo-intensity; IV) CSA and echo-intensity were the quantitative variables most discriminant between asymptomatic players without structural tendon abnormalities and those with tendon abnormalities; V) patellar tendon abnormalities at baseline seem to increase the risk of development of subsequent patellar tendinopathy.

The high prevalence of subclinical tendinopathy and structural tendon abnormalities in the non-dominant side of the investigated group of thrower players can be related to the repetitive overload of the non-dominant lower limb: in fact, the throwing performance of these players is similar to that of javelin throwers who commonly present both subclinical and clinical patellar tendinopathy.

Patellar tendinopathy is commonly evaluated through the ultrasonographic assessment of localized tendon thickening, presence of hypoechoic areas, and altered vascularity $8,13,17$. The two approaches (qualitative and quantitative) we adopted to evaluate the patellar tendons of throwers enabled to assess all these ultrasonographic features: tendon size, tendon echogenicity, and tendon vascularity. The main findings observed in players classified as affected by subclinical tendinopathy (and included in cluster 2) were presence of hypo-echogenic areas and decreased echo-intensity of the non-dominant tendon compared to the dominant one, presence of vessels on Power Doppler analysis, increased thickness and CSA of the non-dominant tendon compared to the dominant one. All these findings represent valid signs of some of the structural changes that occur during the tendinopathic process: increase in tendon thickness, increase in the number of vessels, disorganization of collagen fibers, increase in the hydrated components of the extracellular matrix, and breakdown of tissue organization ${ }^{18-21}$. Other histopatologic findings (that cannot be assessed through ultrasonography) include increase in the number of sensory nerves, proliferation of type III collagen fibers, hypocellularity, increased number of inflammatory cells ${ }^{18-20}$. All these features underlie tendon pathomechanics (i.e., reduced load-bearing capacity) and ultimately result in tendon pain (and increased risk of tendon rupture) ${ }^{18,21}$. 
In the present study, the percentage of structurally abnormal asymptomatic patellar tendons that became symptomatic was $25 \%$. This percentage and the relative risk of 3.89 are in agreement with the results of previous studies 22 indicating that the ultrasonographic structural changes in asymptomatic subjects may represent markers of an early presymptomatic pathology. However, a frequently cited criticism of ultrasound findings is their poor reliability. For instance, ultrasonography is perceived to have a high risk of error when evaluating tendon thickness and $\mathrm{CSA}^{22,23}$. Moreover, the qualitative assessment of Achilles and patellar tendon echogenicity showed poor to moderate inter-observer agreement ${ }^{16}$. On the contrary, quantitative analyses of tendon thickness and CSA showed excellent inter-rater and intra-rater reliability ${ }^{5}$. To our knowledge, this is the first study investigating the longitudinal and transversal echo-intensity in patellar tendons of asymptomatic humans. Previous experimental works assessing the echo-intensity of Achilles tendons in rats $^{24}$ and of infraspinatus tendons in sheeps ${ }^{25}$ found that the echo intensity of the injured tendons was significantly lower compared to intact tendons. Consistently, we observed in the 8 players classified in cluster 2 that both the longitudinal and the transversal echo-intensity were significantly lower in the proximal portion of the left (nondominant) tendon compared to the right (dominant) tendon. Further, we showed that the quantitative variables most discriminant between structurally abnormal and normal patellar tendons were echo-intensity and CSA. Therefore, we recommend the systematic use of these quantitative ultrasound features in crosssectional studies aimed to compare the structural tendon adaptations between different populations ${ }^{26}$ as well as in longitudinal studies aimed to establish the time course of the tendinopathic process ${ }^{8,13}$. It may be hypothesized that the decrease in tendon echo-intensity (that is possibly related to the increase in the hydrated components of the extracellular matrix and to the local inflammatory response) precede the increase in tendon CSA (that is possibly related to disorganization of collagen fibers and proliferation of type III collagen fibers). However, further studies are required to confirm this hypothesis.

There are several limitations to this study. First, the small sample size and the peculiarity of the investigated population of elite throwers make generalization to other (tendons of other) populations of athletes difficult. Second, the short duration of the follow-up (six months) could have implied an underestimation of the risk of development of patellar tendinopathy. Third, the causal association between (ultrasound markers of) tendon histopathology and development of tendinopathy was not demonstrated.

In conclusion, this study showed a high prevalence of abnormalities of the non-dominant patellar tendon in the recruited group of elite players (35\% and $57 \%$ of cases according to the qualitative and quantitative assessments, respectively). Qualitative assessments of tendon structure and neovascularization were in good agreement with the results of the cluster analy- sis applied to quantitative data of CSA and echo-intensity. Finally, we found that patellar tendon abnormalities at baseline seem to increase the risk of development of subsequent patellar tendinopathy.

\section{Conflict of interest}

None.

\section{References}

1. Martinoli C, Derchi LE, Pastorino C, Bertolotto M, Silvestri E. Analysis of echotexture of tendons with US. Radiology. 1993;186:839-843.

2. Rasmussen OS. Sonography of tendons. Scand J Med Sci Sports. 2000;10:360-364.

3. Fredberg U, Bolvig L, Andersen NT, Stengaard-Pedersen K. Ultrasonography in evaluation of Achilles and patella tendon thickness. Ultraschall Med. 2008;29:60-65.

4. Gellhorn AC, Morgenroth DC, Goldstein B. A novel sonographic method of measuring patellar tendon length. Ultrasound Med Biol. 2012;38:719-726.

5. Gellhorn AC, Carlson MJ. Inter-rater, intra-rater, and inter-machine reliability of quantitative ultrasound measurements of the patellar tendon. Ultrasound Med Biol. 2013;39:791-796.

6. Moustafa AM, Hassanein E, Foti C. Objective assessment of corticosteroid effect in plantar fasciitis: additional utility of ultrasound. Muscles Ligaments Tendons J. 2016;5:289-296.

7. Kulig K, Landel R, Chang YJ, Hannanvash N, Reischl SF, Song P, Bashford GR. Patellar tendon morphology in volleyball athletes with and without patellar tendinopathy. Scand J Med Sci Sports. 2013;23:e81-88.

8. Giombini A, Dragoni S, Di Cesare A, Di Cesare M, Del Buono A, Maffulli N. Asymptomatic Achilles, patellar, and quadriceps tendinopathy: a longitudinal clinical and ultrasonographic study in elite fencers. Scand J Med Sci Sports. 2013;23:311316.

9. Comin J, Cook JL, Malliaras P, McCormack M, Calleja M, Clarke A, Connell D. The prevalence and clinical significance of sonographic tendon abnormalities in asymptomatic ballet dancers: a 24-month longitudinal study. Br J Sports Med. 2013:47:89-92.

10. Visnes $\mathrm{H}$, Tegnander A, Bahr R. Ultrasound characteristics of the patellar and quadriceps tendons among young elite athletes. Scand J Med Sci Sports. 2015;25:205-215.

11. Van der Worp H, de Poel HJ, Diercks RL, van den AkkerScheek I, Zwerver J. Jumper's knee or lander's knee? A systematic review of the relation between jump biomechanics and patellar tendinopathy. Int J Sports Med. 2014;35:714-722.

12. Reinking MF. Current concepts in the treatment of patellar tendinopathy. Int J Sports Phys Ther. 2016;11:854-866.

13. Cook JL, Khan KM, Kiss ZS, Coleman BD, Griffiths L. Asymptomatic hypoechoic regions on patellar tendon ultrasound: A 4year clinical and ultrasound follow-up of 46 tendons. Scand J Med Sci Sports. 2001;11:321-327.

14. Elias LJ, Bryden MP, Bulman-Fleming MB. Footedness is a better predictor than is handedness of emotional lateralization. Neuropsychologia. 1998;36:37-43.

15. Padulo J, Oliva F, Frizziero A, Maffulli N. Muscles, Ligaments and Tendons Journal - Basic principles and recommendations in clinical and field science research: 2016 update. MLTJ. 2016;6(1):1-5.

16. Sunding K, Fahlström M, Werner S, Forssblad M, Willberg L. Evaluation of Achilles and patellar tendinopathy with greyscale ultrasound and colour Doppler: using a four-grade scale. Knee 
Surg Sports Traumatol Arthrosc. 2016;24:1988-1996.

17. Ooi CC, Richards PJ, Maffulli N, Ede D, Schneider ME, Connell $D$, Morrissey $D$, Malliaras $P$. A soft patellar tendon on ultrasound elastography is associated with pain and functional deficit in volleyball players. J Sci Med Sport. 2016;19:373-378.

18. Sharma P, Maffulli N. Biology of tendon injury: healing, modeling and remodeling. J Musculoskelet Neuronal Interact. 2006;6:181-190.

19. Khan KM, Maffulli N, Coleman BD, Cook JL, Taunton JE. Patellar tendinopathy: some aspects of basic science and clinical management. Br J Sports Med. 1998;32:346-355.

20. Khan KM, Cook JL, Bonar F, Harcourt P, Astrom M. Histopathology of common tendinopathies. Update and implications for clinical management. Sports Med. 1999;27:393-408.

21. Scott A, Backman LJ, Speed C. Tendinopathy: Update on Pathophysiology. J Orthop Sports Phys Ther. 2015;45:833841.
22. McAuliffe S, McCreesh K, Culloty F, Purtill H, O'Sullivan K. Can ultrasound imaging predict the development of Achilles and patellar tendinopathy? A systematic review and metaanalysis. Br J Sports Med. 2016;50:1516-1523.

23. Ekizos A, Papatzika F, Charcharis G, Bohm S, Mersmann F, Arampatzis A. Ultrasound does not provide reliable results for the measurement of the patellar tendon cross sectional area. J Electromyogr Kinesiol. 2013;23:1278-1282.

24. Chamberlain CS, Duenwald-Kuehl SE, Okotie G, Brounts SH, Baer GS, Vanderby R. Temporal healing in rat achilles tendon: ultrasound correlations. Ann Biomed Eng. 2013;41:477-487.

25. Frisch KE, Marcu D, Baer GS, Thelen DG, Vanderby R. Influence of tendon tears on ultrasound echo intensity in response to loading. J Biomech. 2014;47:3813-3819.

26. Malliaras P, Kamal B, Nowell A, Farley T, Dhamu H, Simpson V, Morrissey D, Langberg H, Maffulli N, Reeves ND. Patellar tendon adaptation in relation to load-intensity and contraction type. J Biomech. 2013;46:1893-1899. 